\title{
Fabrication and Characterization of a High-Gain Yb-Er Codoped Phosphate Glass Optical Amplifier
}

\author{
Gerardo Cristian Scarpignato, ${ }^{1}$ Daniel Milanese, ${ }^{1}$ Joris Lousteau, ${ }^{2}$ \\ Nadia Giovanna Boetti, ${ }^{1}$ and Emanuele Mura ${ }^{1}$ \\ ${ }^{1}$ DISAT, Politecnico di Torino, Corso Duca degli Abruzzi 24, 10129 Torino, Italy \\ ${ }^{2}$ PhotonLab, Istituto Superiore Mario Boella, Via P. Boggio 61, 10138 Torino, Italy \\ Correspondence should be addressed to Gerardo Cristian Scarpignato; gerardo.scarpignato@polito.it
}

Received 31 January 2013; Accepted 23 April 2013

Academic Editor: Oualid Hammi

Copyright (C) 2013 Gerardo Cristian Scarpignato et al. This is an open access article distributed under the Creative Commons Attribution License, which permits unrestricted use, distribution, and reproduction in any medium, provided the original work is properly cited.

\begin{abstract}
We present the fabrication and characterization of a short core pumped fiber amplifier made of $\mathrm{Yb}^{3+}-\mathrm{Er}^{3+}$ codoped phosphate glasses. The $27 \mathrm{~mm}$ long amplifier provides a maximum internal gain of $10.7 \mathrm{~dB}$ for a signal of $-30 \mathrm{dBm}$ at $1535 \mathrm{~nm}$ with a $479 \mathrm{~mW}$ pump power operating at $975.6 \mathrm{~nm}$. This leads to a gain of $4.0 \mathrm{~dB} / \mathrm{cm}$, which compares favorably to other recent works.
\end{abstract}

\section{Introduction}

Thanks to the high spatial and spectral quality of their output beam, fiber lasers appear as a promising infrared light source for LIDAR systems. As fiber laser material, phosphate glasses allow to incorporate high levels of rareearth ions, which enables the manufacturing of short optical fiber devices with a high output power. These features redound into compact, portable lasers that can reach longer distances when compared with other emitting devices. Usually, a one-piece fiber laser having all these characteristics cannot be easily conceived. A convenient way to achieve all the specifications at once is to proceed through a master oscillator power amplifier (MOPA) architecture. In this configuration, the output of a low-power seed laser having the required spectral characteristics is carried into a high-power fiber amplifier. This, in turn, provides the required amplification level without impairing on the other desired specification. This paper focuses precisely on the fabrication and characterization of a short core pumped fiber amplifier based on $\mathrm{Yb}^{3+}-\mathrm{Er}^{3+}$ codoped phosphate glasses to be used at the second stage of a MOPA configuration.

\section{Experimental Details}

2.1. Optical Fiber Fabrication and Characterization. The core glass was doped with $2.81 \mathrm{wt} \%$ of $\mathrm{Er}_{2} \mathrm{O}_{3}\left(1.08 \mathrm{~mol} \%, 2.5 \cdot 10^{26}\right.$ ions $\left./ \mathrm{m}^{3}\right)$ and $2.87 \mathrm{wt} \%$ of $\mathrm{Yb}_{2} \mathrm{O}_{3}\left(1.09 \mathrm{~mol} \%, 2.5 \cdot 10^{26}\right.$ ions $/ \mathrm{m}^{3}$ ). The optical fiber preform was produced by means of rotational casting technique developed in-house. The preform and fiber fabrication parameters were set to generate a double-cladding fiber with the following diameters: $7 \mu \mathrm{m}$, $25 \mu \mathrm{m}$, and $70 \mu \mathrm{m}$ for the core, inner, and outer cladding, respectively. The preform was drawn into fiber using a drawing tower developed in-house. The core diameter was chosen to provide an acceptable coupling efficiency to the commercial Flexcore fibers used in the system. The double cladding topology allows us to test the fiber as either core or cladding pumped; the latter case will be addressed elsewhere. The refractive indexes of the core and inner cladding were designed in order to obtain a numerical aperture $\mathrm{NA}=0.17$. This value guarantees that the active fiber presents an almost single-mode behavior at the signal wavelength. The quality and morphology of the optical fiber were inspected by means of a Nikon ECLIPSE E 50i microscope. The active fiber loss measurements were obtained by the cut-back technique using 
a single-mode fiber laser diode operating at $1.3 \mu \mathrm{m}$. The laser was butt coupled to one of the active fiber ends. A set of nearfield images were taken at the other end of a $280 \mathrm{~cm}$ long fiber section in order to analyze the modal properties.

2.2. Amplifier Gain Experiments. The components used in the experimental setup are summarized in Table 1. A singlemode laser diode working at $975.6 \mathrm{~nm}$ with a maximum power of around $500 \mathrm{~mW}$ was used for the pump. A tunable laser ranging from 1500 to $1600 \mathrm{~nm}$ with output power levels from $-30 \mathrm{dBm}$ up to $+5 \mathrm{dBm}$ was used for the signal. Both components were spliced to the corresponding inputs of a wavelength division multiplexer (WDM). Its output was butt coupled to the active fiber, which consists of a $27 \mathrm{~mm} \mathrm{Yb}^{3+} / \mathrm{Er}^{3+}$ codoped phosphate glass fiber. All these components except for the amplifier section use Flexcore 1060 fibers with $5.6 \mu \mathrm{m}$ diameter. This ensures a correct matching among them. A photograph of the in-house fabricated active fiber is shown in Figure 1. A pigtail with $9 \mu \mathrm{m}$ core diameter was used to convey the active fiber output power to the optical spectrum analyzer (OSA) in order to measure the output power of the amplified signal.

\section{Results}

3.1. Optical Fiber Modal Properties. The near-field image of the active fiber end face at $1300 \mathrm{~nm}$ is presented in Figure 2(a). The transversal profile is depicted in Figure 2(b). As can be observed, the power is almost entirely confined inside the core. A slightly diffused area corresponding to evanescent wave can barely be seen inside the inner cladding. The confinement ensures that the pump power is not irradiated outside the active area and therefore it is available to excite the active ions with which the signal can interact. While adjusting the launching condition, the presence of a profile similar to the $\mathrm{LP}_{11}$ mode could be observed. This observation confirms the multimode behavior of the core at $1300 \mathrm{~nm}$.

3.2. Optical Fiber Attenuation Measurements. Fiber losses were measured by cut-back technique using a length of $280 \mathrm{~cm}$ with a source at $1300 \mathrm{~nm}$. The results are depicted in Figure 3. The fitted attenuation coefficient was $7.11 \mathrm{~dB} / \mathrm{m}$. Measurements data show a deviation with respect to the fitted slope. This deviation can be attributed to experimental errors caused by several factors such as the hand cleaving procedure, the presence of scattering particles, or short range changes on the fiber diameter.

3.3. Optical Fiber Amplifier Demonstration. The output spectrum of the amplifier is shown in Figure 4 after the fiber had been excited with a $+0 \mathrm{dBm}$ signal at $1535 \mathrm{~nm}$ and $479 \mathrm{~mW}$ of pump power. It is worth mentioning that a spurious laser peak at $1534 \mathrm{~nm}$ could be observed on the OSA during the experiments when refractive index matching gel was not applied at the coupling ends of the phosphate glass fiber with the launching and exiting commercial silica glass fibers. For that reason, all measurements reported in this paper were carried out using index matching gel. Not only did the gel
TABLE 1: Material and components.

\begin{tabular}{|c|c|c|}
\hline Component & Value/description & Relevant parameters \\
\hline Pump laser & $\begin{array}{l}\text { 1999CHP } \\
\text { (3SGroup) } \\
\text { HI1060 fiber output }\end{array}$ & $\begin{array}{l}975.6 \mathrm{~nm} \\
650 \mathrm{~mW}(\max ) \\
\varnothing 5.6 \mu \mathrm{m} / 0.125 \mathrm{NAPM}\end{array}$ \\
\hline Tunable laser & HP8168F & $\begin{array}{l}\lambda>1500 \mathrm{~nm} \\
\lambda<1600 \mathrm{~nm}\end{array}$ \\
\hline WDM & $\begin{array}{l}\text { Go4fiber } \\
\text { WDM9890-250-A-1 }\end{array}$ & $\begin{array}{l}\text { Ports: } 976-1090 \mathrm{~nm} \\
26 \mathrm{~dB} \text { isolation } \\
\text { Fiber } \varnothing=5.6 \mu \mathrm{m} \mathrm{SM}\end{array}$ \\
\hline $\begin{array}{l}\mathrm{Yb}^{3+} / \mathrm{Er}^{3+} \\
\text { fiber }\end{array}$ & $\begin{array}{l}\text { In-house developed } \\
\text { phosphate glass fiber }\end{array}$ & $\begin{array}{l}\text { Fiber } \emptyset_{\text {core }}=7 \mu \mathrm{m} \\
\text { Fiber } \emptyset_{\text {innerclad }}=25 \mu \mathrm{m} \\
\mathrm{NA}=0.17\end{array}$ \\
\hline $\begin{array}{l}\text { Collector } \\
\text { pigtail }\end{array}$ & & $\begin{array}{l}\text { Fiber } \emptyset=9 \mu \mathrm{m}(\mathrm{SM}) \\
\text { Fiber } \emptyset_{\text {clad }}=125 \mu \mathrm{m}\end{array}$ \\
\hline OSA & Agilent 86140B & $\begin{array}{l}\text { Max } 30 \mathrm{dBm} \text { or } \\
23 \mathrm{dBm} \text { in } 10 \mathrm{~nm}\end{array}$ \\
\hline
\end{tabular}

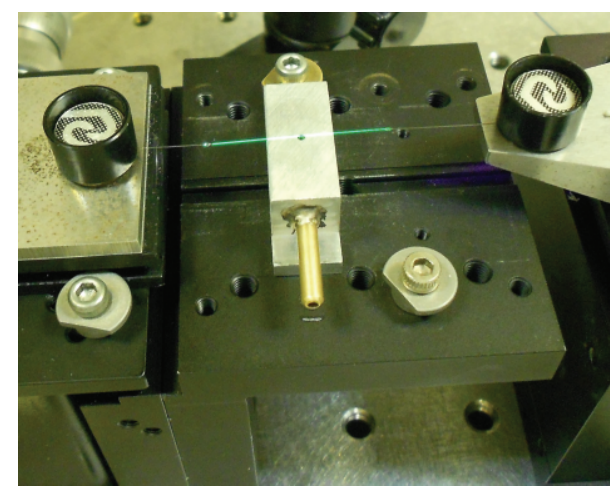

FIGURE 1: A detailed view of the fabricated amplifier section.

improve the coupling efficiency between butt-coupled fibers but it also because contributed to the mechanical stability of the measurement by maintaining the fibers correctly faced one to another and by avoiding spurious movements produced by small air fluctuations.

3.4. Optical Fiber Gain Measurements. The presence of green light scattered out of the fiber-as seen in Figure 1-indicates that the pumping efficiency is limited by upconversion processes. It also increased with increasing pump power. Moreover, the losses introduced by the fiber couplings in the input-output path have been evaluated to be between 4.52 and $5.17 \mathrm{~dB}$. Additionally, since the WDM was designed to work with $1090 \mathrm{~nm}$ signals, the losses that the device exerts on injected signals of other wavelengths ranging from 1510 to $1590 \mathrm{~nm}$ had to be evaluated. The values found were between $0.65 \mathrm{~dB}$ and $0.47 \mathrm{~dB}$, almost independently of the signal power used. Although these losses were present, the amplifier showed net gain (see Figure 5) for signal wavelengths ranging from $1527 \mathrm{~nm}$ up to $1549 \mathrm{~nm}$ and signal powers from $-30 \mathrm{dBm}$ up to $0 \mathrm{dBm}$. The net gain reached a maximum level of $4.7 \mathrm{~dB}$ at $1534 \mathrm{~nm}$. The internal gain was also evaluated taking into account the coupling losses. In order to provide the most 


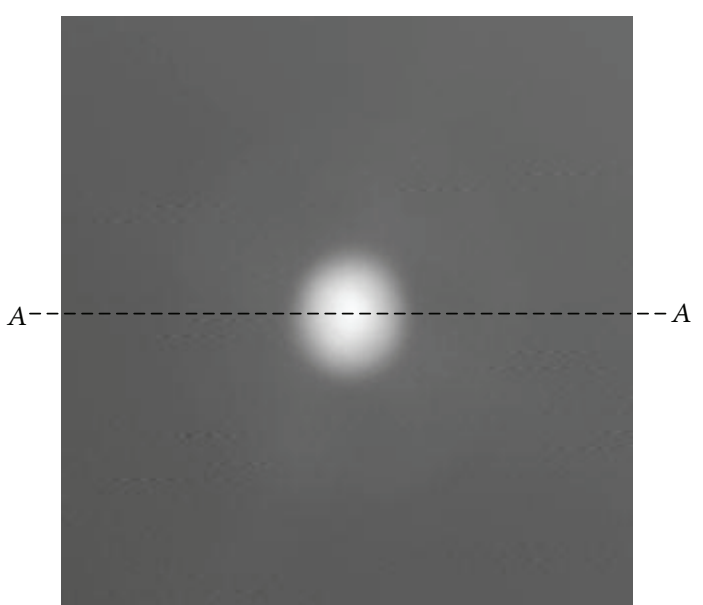

(a)

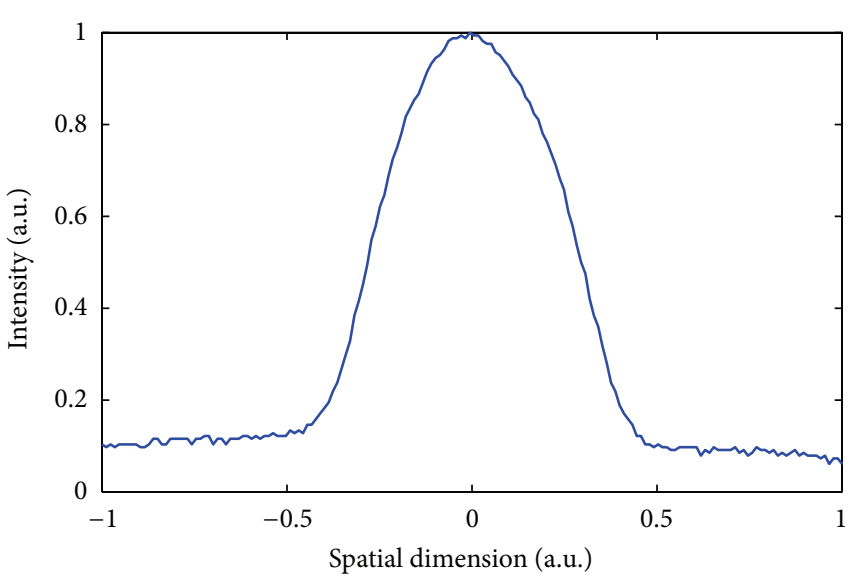

(b)

Figure 2: (a) Near-field image of the active fiber end at $1300 \mathrm{~nm}$. (b) Intensity profile corresponding to the transversal dashed line $A$ - $A$ shown in (a).

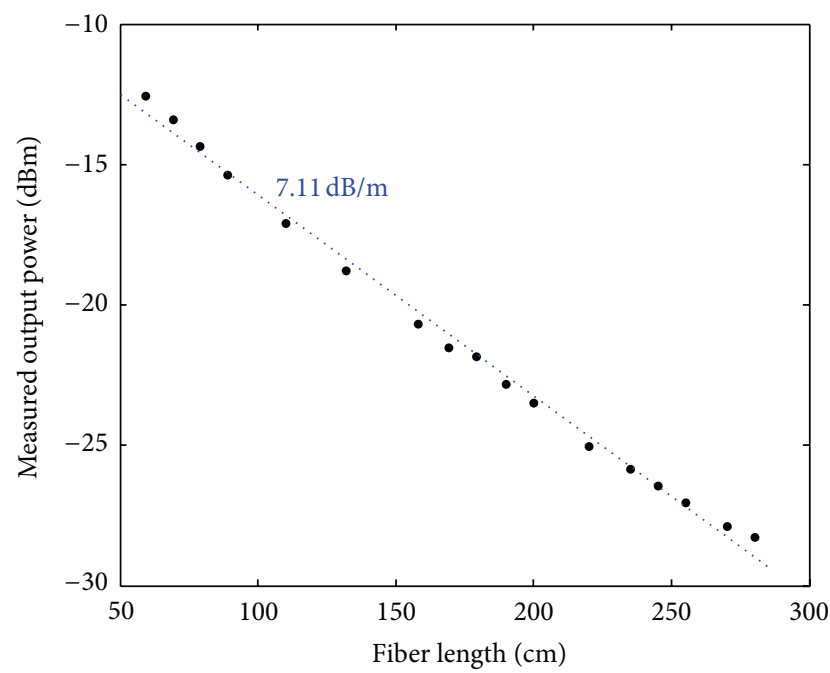

Figure 3: Attenuation coefficient measured on $\mathrm{Yb}^{3+} / \mathrm{Er}^{3+}$ phosphate glass fiber obtained by exciting at $1300 \mathrm{~nm}$. After fitting the curve, a mean value of $7.11 \mathrm{~dB} / \mathrm{m}$ was found.

conservative value for the internal gain in all the cases, it was adopted a value of $4 \mathrm{~dB}$ for the coupling losses. As a result, it can be deduced that the active medium provides amplification for a wider range of wavelengths, extending from $1515 \mathrm{~nm}$ to $1575 \mathrm{~nm}$. In this set of measurements, the fiber provided an internal gain as high as $8.7 \mathrm{~dB}$ for a $-20 \mathrm{dBm}$ signal.

The internal gain per unit length at wavelength $\lambda=$ $1535 \mathrm{~nm}$ is presented in Figure 6. It presents gain value between 1 and $4 \mathrm{~dB}$ depending on the available pump power. As discussed in Section 3.3 and despite all the efforts that have been taken, errors related to stability issues impaired slightly some of the measurements. This is evident in Figure 6 where the gain curves tend to be closer one to each other. Due to the experimental errors, lower pump powers produce higher

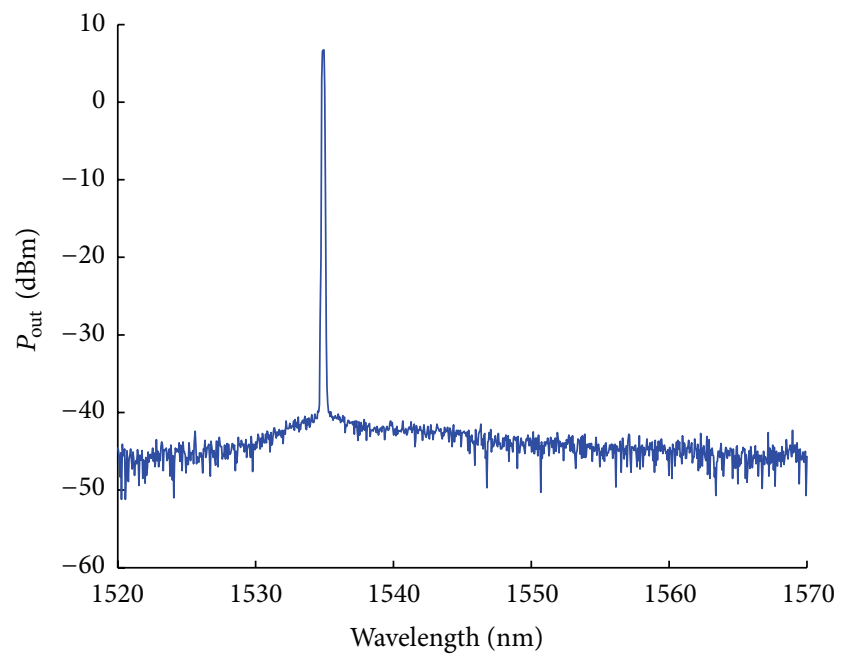

FIGURE 4: Amplifier output spectrum of the $\mathrm{Yb}^{3+} / \mathrm{Er}^{3+}$ phosphate glass fiber obtained by exciting with a $479 \mathrm{~mW}$ pump at $975 \mathrm{~nm}$ and a $0 \mathrm{dBm}$ signal at $1535 \mathrm{~nm}$. The highest peak obtained at these conditions is shown $(+6.714 \mathrm{dBm})$.

gain results when it should be the contrary. For instance, the gain for a signal of $-20 \mathrm{dBm}$ at a $378.2 \mathrm{~mW}$ pump power is higher than for a pump power $479.8 \mathrm{~mW}$. The same situation occurs for a $+5 \mathrm{dBm}$ at $237.7 \mathrm{~mW}$.

A maximum value of $4.0 \mathrm{~dB} / \mathrm{cm}$ was obtained for a $-30 \mathrm{dBm}$ signal and $479.8 \mathrm{~mW}$ pump power. Here, the curves show that the amplifier presents a clear sign of saturation at high pump or signal powers. This is in agreement with the fact that further increments in the pump power do not have a corresponding linear increase on the internal gain. At low pump conditions, the internal gain levels maintain almost a constant value independent of the input signal power (at least for the range of tested signal powers). This observation suggests that, in this regime, the $\mathrm{Er}^{3+}$ is not yet fully inverted 


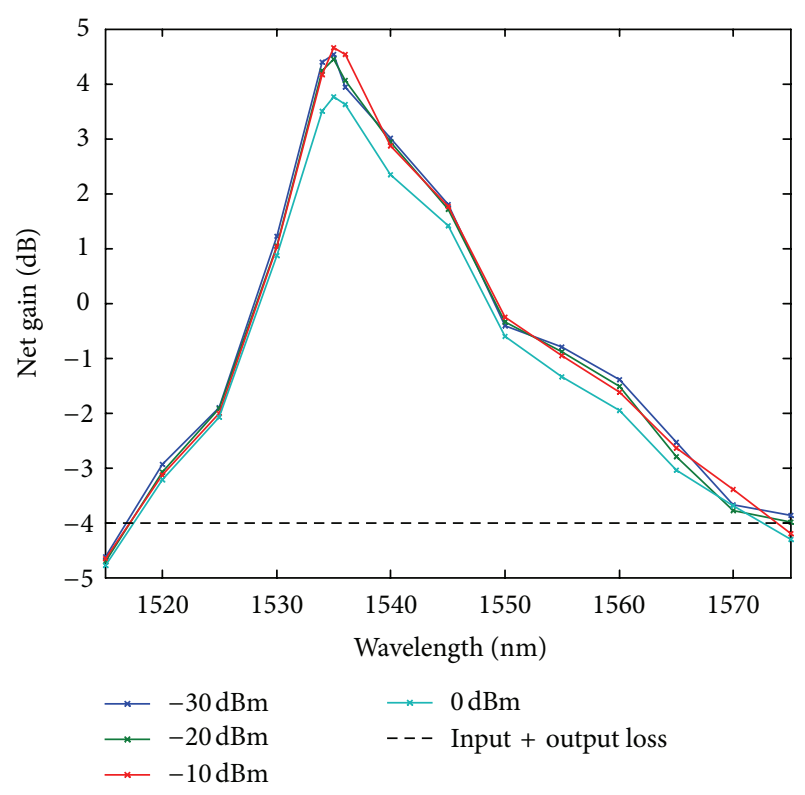

FIGURE 5: Signal net gain spectrum obtained for four different signal input powers $-30 \mathrm{dBm},-20 \mathrm{dBm},-10 \mathrm{dBm}$, and $0 \mathrm{dBm}$ at wavelengths ranging from 1515 to $1575 \mathrm{~nm}$. Injected pump power is $P_{p}=237 \mathrm{~mW}$.

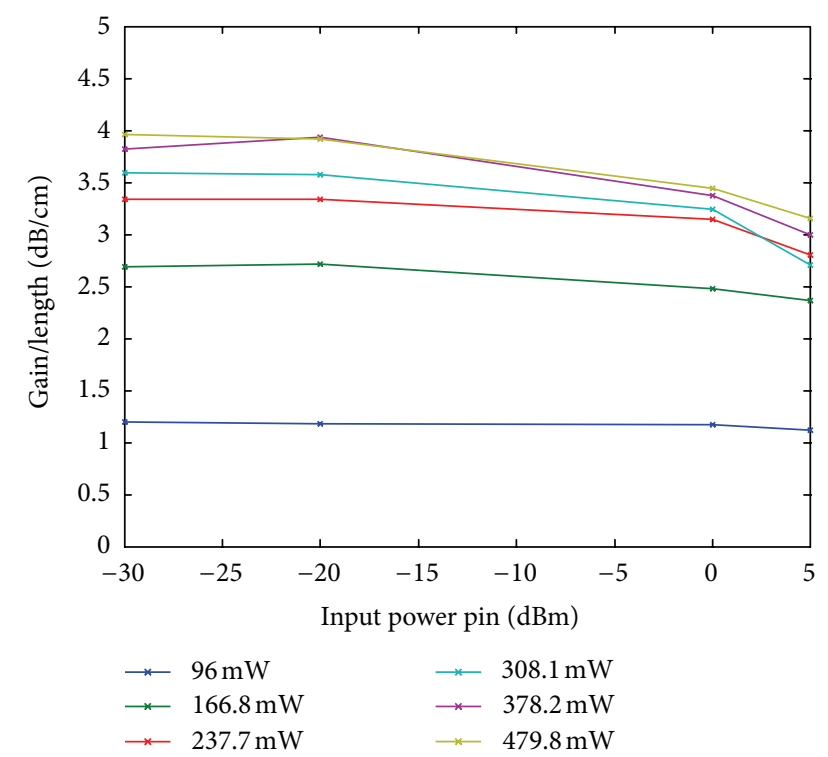

FIGURE 6: Internal gain per unit length as a function of the input power level at $1535 \mathrm{~nm}$. The amplifier shows some degree of saturation.

and that the amplifier is not yet saturated. When increasing the pump power, the gain starts to saturate for both low and high signal input cases. However, one can notice that the saturation gain tends to decrease as the input signal increases. Actually, deeming the amplifier as a power reservoir, this latter is not large enough to sustain the same gain value for high input signal as it does for low signal input power. It is believed that in order to increase the gain per length value a further improvement of the fabrication process is required. For instance, the reduction of particles and defects located at the core-cladding interface would allow reducing the optical loss and thus achieving more gain. Additionally, avoiding the creation of micro-clusters could be useful to reduce upconversion in order to increase the output power.

Also, at high pump power a large amount of pump remained unabsorbed and was in fact observed experimentally at the amplifier output. An amplifier slightly longer would allow to fully exploit the pump power available for reaching higher output powers.

\section{Conclusions}

A fiber amplifier based on $\mathrm{Yb}^{3+}-\mathrm{Er}^{3+}$ codoped phosphate glasses was fabricated and characterized. At the highest input signal $(+5 \mathrm{dBm})$ the amplifier provided $6.39 \mathrm{~dB}$ and $8.52 \mathrm{~dB}$ internal gain for medium and highly saturated operation (166.8 $\mathrm{mW}$ and $479.8 \mathrm{~mW}$ pump power, resp.). The internal gain per unit length was thus $2.37 \mathrm{~dB} / \mathrm{cm}$ and $3.15 \mathrm{~dB} / \mathrm{cm}$, which is higher than or near to values reported in $[1,2]$ $(0.94 \mathrm{~dB} / \mathrm{cm}$ and $3.3 \mathrm{~dB} / \mathrm{cm})$. In addition, the maximum observed internal gain was $10.71 \mathrm{~dB}$, obtained with the minimum input signal $(-30 \mathrm{dBm})$ and a $479 \mathrm{~mW}$ pump power. This leads to an internal gain of $4.0 \mathrm{~dB} / \mathrm{cm}$, a value that is comparable to the values reported in $[3,4](4.2 \mathrm{~dB} / \mathrm{cm}$ and $5.2 \mathrm{~dB} / \mathrm{cm}$, resp.).

\section{References}

[1] W. Shi, M. Leigh, J. Zong, Z. Yao, and S. Jiang, "Photonic narrow linewidth GHz source based on highly codoped phosphate glass fiber lasers in a single MOPA chain," IEEE Photonics Technology Letters, vol. 20, no. 2, pp. 69-71, 2008.

[2] S. H. Xu, Z. M. Yang, Z. M. Feng, Q. Y. Zhang, Z. H. Jiang, and W. C. Xu, "Gain and noise characteristics of single-mode $\mathrm{Er}^{3+} / \mathrm{Yb}^{3+}$ Co-doped phosphate glass fibers," in Proceedings of the 2nd IEEE International Nanoelectronics Conference (INEC '08), pp. 633635, March 2008.

[3] Z. Pan, Q. Xu, L. Meng, H. Cai, Z. Fang, and R. Qu, "Singlepolarization operation of phosphate fiber laser induced by external feedback," in Proceedings of the 8th Pacific Rim Conference on Lasers and Electro-Optics (CLEO/Pacific Rim '09), p. 1249, September 2009.

[4] S. H. Xu, Z. M. Yang, T. Liu et al., "An efficient compact $300 \mathrm{~mW}$ narrow-linewidth single frequency fiber laser at $1.5 \mathrm{um}$," Optics Express, vol. 18, no. 2, pp. 1249-1254, 2010. 

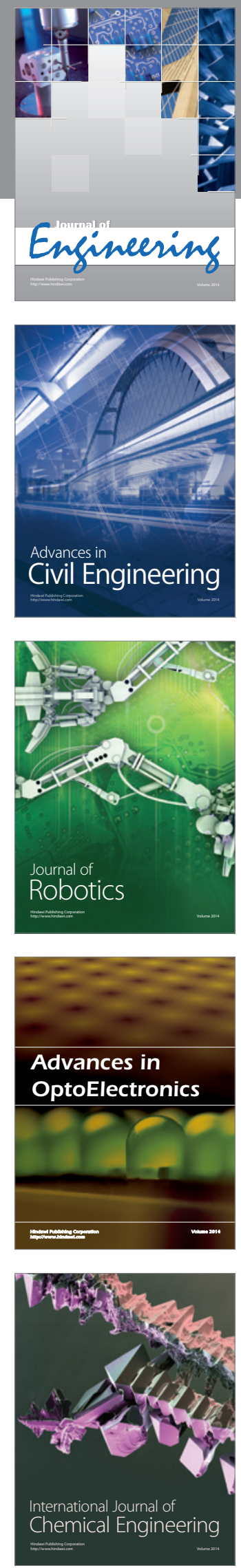

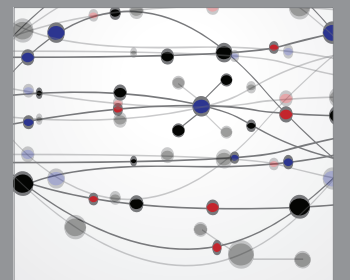

The Scientific World Journal
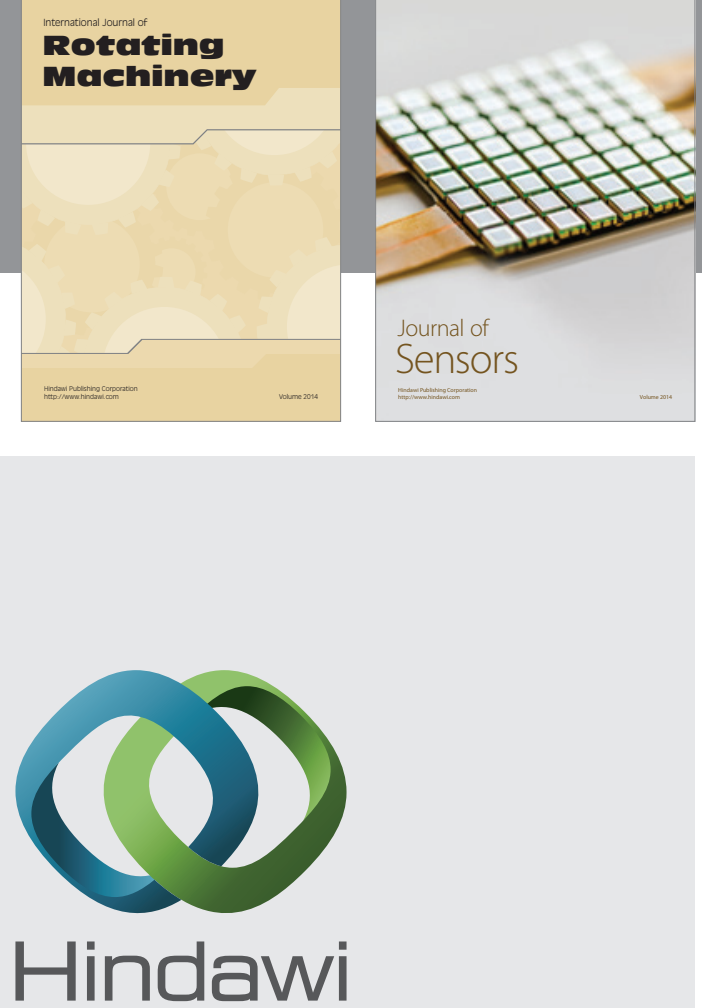

Submit your manuscripts at http://www.hindawi.com
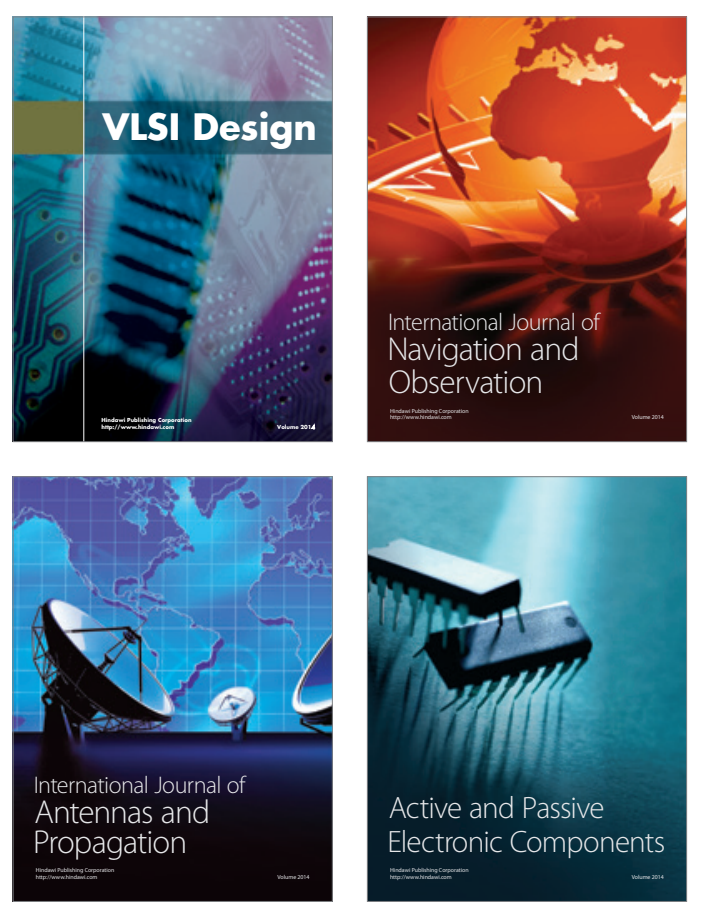
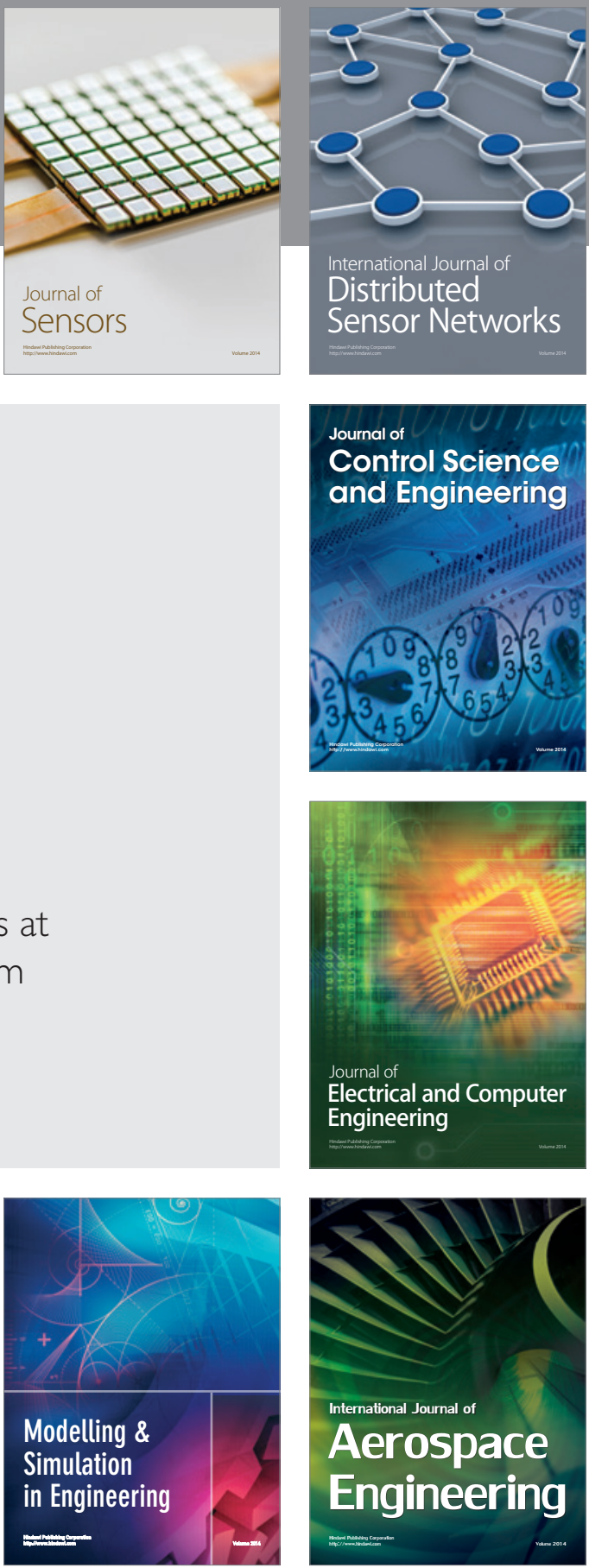

Journal of

Control Science

and Engineering
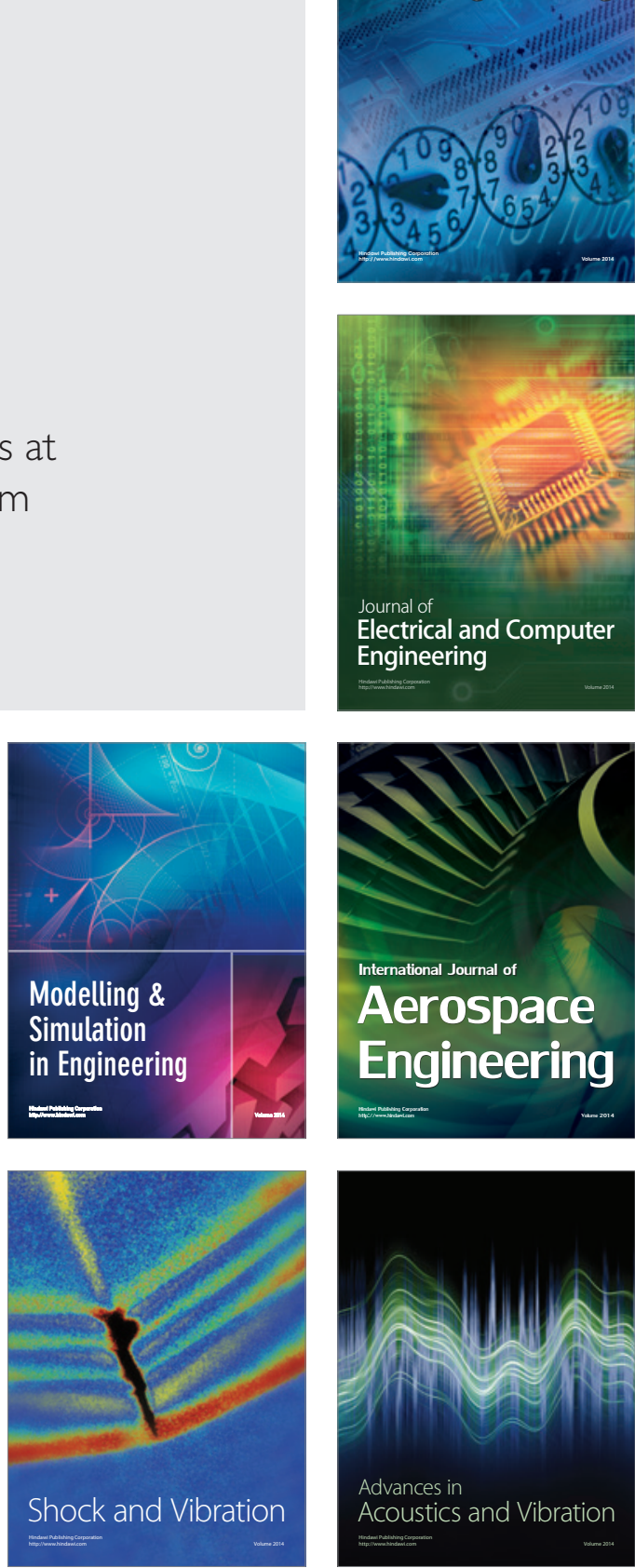